09 'IT'S NOT ANOTHER DIET': BARIATRIC DIETITIANS' ALTERNATIVE TALK ABOUT FOOD AND THE BODY

Hilla Nehushtan. Hebrew University of Jerusalem, Jerusalem, Israel

10.1136/bmjopen-2019-QHRN.9

Background Themes of surveillance and control in the context of weight loss (bariatric) surgery are well established in existing critical literature, usually contrasted with joy and pleasure. Nonetheless, researchers have recently disrupted this dichotomy and showed how some clinicians use more empathic, enjoyable methods of weight loss.

This research continues this disruption and states that bariatric dietitians offer different perspectives on food behavior and of the body than those of popular biomedical approaches. While this 'anti-diet' discourse is not limited to bariatrics, I argue that for bariatric patients this new discourse is liberating in many ways.

Methods This paper is based on interviews with 5 bariatric dietitians and on participant observation in a weight loss clinic: I joined 55 dietitian-patient consultation sessions and 20 meetings of a bariatric support group, guided by a dietitian and a psychotherapist.

Findings Bariatric dietitians state that the surgery and guidelines that follow it are part of a new worldview. They construct the post-surgery condition as a processual, relational, ongoing individual path, and evaluate the success of the surgery as a function of maintaining healthy eating and an active lifestyle, as opposed to meticulously counting calories and tracking numbers on the scale. They aim to reduce shame and blame resulting from eating behavior and actively object to 'diet talk' - phrases describing certain foods as 'prohibited'.

The patients themselves take pride in this new agenda which distances them from the two extremes of food behavior: they are no longer the out-of-control eaters they saw themselves to be prior to the surgery, yet also avoid being the over controlling dieters, who exercised unhealthy weight loss methods. In this sense, they exemplify the meaningful change not only of their body, but also of their new self.

\section{COMMUNITY HEALTH WORKERS: SOLVING HEALTH ISSUES AND IMPROVING WELLBEING}

Ryan Logan. University of South Florida, Tampa, USA

\subsection{6/bmjopen-2019-QHRN.10}

As the U.S. health care system continues to face a myriad of challenges due to political uncertainty, cost, lack of reform, and financial insecurity, many individuals and communities throughout the U.S. will continue to encounter barriers to accessing care. Among the most vulnerable groups include black, immigrant, refugee, and low-income communities. These groups encounter significant barriers in the form of social determinants of health - such as lack of financial security, education level, and transportation. One type of non-clinical worker, commonly known as a community health worker $(\mathrm{CHW})$, provides one means of addressing these issues. However, in many states, CHWs are not well integrated within the workforce and/or are treated with ambivalence. Their strengths include belonging to the community they work within, emphasizing community outreach, health education, and advocacy.
Their strengths address vital gaps in care for marginalized communities.

This paper explores key findings from a dissertation project that documented the lived experiences of CHWs. The aim of this paper is to present the practical and theoretical findings regarding these workers as they are integrated into the workforce in the state of Indiana. The theoretical framing of this project includes moral economy and anthropological framings of health care. The project utilized a collaborative approach with a local CHW organization and drew on participant observation ( $>300$ hours), semi-structured interview ( $\mathrm{n}=50$ interviews), focus groups, and photovoice. The results demonstrate similar yet diverse experiences among these workers, disparities in the vision of the CHW model between stakeholders and CHWs, and the vital role of advocacy in promoting health and wellbeing. Overall, this project demonstrates that social scientists can play a crucial role in infusing social theory in conceptions of health and wellbeing in addition to fostering collaborative research relationships with community partners.

\section{INTEGRATING NEW BIOMEDICAL TECHNOLOGIES IN IVF TREATMENT: THE CASE OF TIME-LAPSE IMAGING}

Manuela Perrotta, Alina Geampana, Josie Hamper. Queen Mary University of London, London, UK

\subsection{6/bmjopen-2019-QHRN.11}

Time-lapse imaging has recently been introduced in fertility treatment and represents a controversial, yet potentially revolutionary tool for IVF practice. Time-lapse technologies take pictures of embryos at regular intervals (every 5, 10, or $20 \mathrm{~min}$ ) and produce a large amount of images for each embryo. This data, in conjunction with computer algorithms, is meant to help professionals pick the best embryo to be implanted. Embryo selection is essential in IVF practice for increasing pregnancy rates and reducing the negative effect of repeated failures. Nonetheless, a significant number of clinics charge additional fees for this new, yet clinically unproven technology. Stemming from a larger research project that investigate the case of time-lapse monitoring, this paper's objective is to analyse the emergence of a new medical technology, focusing on its reception by professionals and the public alike. Our research asks: How is time-lapse technology currently integrated in clinical practice? How is it advertised to patients? How is it perceived outside professional circles? Using ethnographic data collected from 5 UK clinical sites (observations in IVF labs and interviews with professionals) and document analysis (medical literature, manufacturers' and clinics' websites, public documents produced by stakeholders), we argue that the case of time-lapse reveals several shortcomings in the development and uptake of new technologies in IVF practice. More specifically, we critically explore three inter-related areas that have influenced time-lapse use in UK clinics: patient expectations, IVF marketing and technology trends, and IVF professionals' integration of technological innovation in medical practice. We conclude that the development, use and marketing of new medical technologies must be sensitive to the perpetuation of inequities in health access. The use and cost associated with time-lapse technology is important in shaping inequalities of access to health care services in the global fertility market. 